\title{
Development of WHO Guidelines on Physical Activity, Sedentary Behavior, and Sleep for Children Less Than 5 Years of Age
}

\author{
Juana Willumsen and Fiona Bull
}

\begin{abstract}
Background: Physical inactivity is a leading risk factor for global mortality and a contributor to the increase in overweight and obesity. The Commission on Ending Childhood Obesity identified the need for guidance on physical activity, particularly for early childhood $(<5 \mathrm{y})$, a period of rapid physical and cognitive development. Methods: The World Health Organization (WHO) has developed the first global guidelines on physical activity, sedentary, and sleep behaviors, building upon high-quality systematic reviews. The WHO guideline process is a rigorous, systematic, and transparent method for the development of recommendations, using the Grading of Recommendations Assessment, Development and Evaluation Evidence to Decision framework. It takes into consideration the strength of the evidence as well as values and preferences, benefits and harms, equity and human rights. Results: The authors summarize the first global guidelines on time spent in physical activity, sedentary behavior (including screen time and time spent restrained), and sleep patterns in infants (birth to $1 \mathrm{y}$ of age), toddlers (1-2.9 y of age), and preschoolers (3-4.9 y of age). Conclusions: WHO is actively disseminating and supporting implementation of these guidelines by national adoption and adaptation, through links with early childhood development and the Global Action Plan on Physical Activity 2018-2030.
\end{abstract}

Keywords: young children, movement, recommendations

Physical inactivity has been identified as a leading risk factor for global mortality and a contributor to the increase in overweight and obesity. Although we know that more than $28 \%$ of adults ${ }^{1}$ and $80 \%$ of adolescents are not sufficiently physically active, ${ }^{2}$ there are currently no comparable global data for younger children.

Global recommendations on physical activity for health have been established for 3 population age groups ${ }^{3}(5-17$ y, 18-64 y, and $65 \mathrm{y}$ and older), but prior to 2019 , the recommendation did not include children less than 5 years. Early childhood $(<5 \mathrm{y})$ is a period of rapid physical and cognitive development, and this is the time period during which a child's habits are formed and family lifestyle routines are open to changes and adaptations. The importance of interactions among physical activity, sedentary behavior, and adequate sleep time on physical and mental health and well-being were recognized by the Commission on Ending Childhood Obesity, which called for clear guidance on physical activity, sedentary behavior, and sleep in young children. ${ }^{4}$ By establishing such guidelines, it will be possible to conduct population surveillance of movement behaviors against recommendations, monitor trends over time and inequalities, and provide evidence to inform interventions. These specific guidelines are necessary to address the childhood obesity epidemic globally. As healthy physical activity, sedentary behavior, and sleep habits are established early in life, these guidelines provide an opportunity to shape these habits through childhood, adolescence, and into adulthood. ${ }^{5}$ Developing healthy lifestyle habits and changing social norms to support increased physical activity throughout the life course are key elements of the Global Action Plan on Physical Activity, ${ }^{6}$ which aims to reduce physical inactivity by $15 \%$ by 2030 . By establishing recommendations for young children, this will enable adherence to

The authors are with the World Health Organization, Geneva, Switzerland. Willumsen (willumsenj@who.int) is corresponding author. these to be monitored, to build a full picture of the behaviors contributing to children's healthy development.

This study summarizes the process by which World Health Organization (WHO) developed guidelines on physical activity, sedentary, and sleep behaviors, as requested by the Commission on Ending Childhood Obesity. The WHO guideline process is a rigorous, systematic, and transparent process for the development of recommendations that takes into consideration the strength of the evidence as well as values and preferences, benefits and harms, equity and human rights.

\section{Methods}

\section{Scope and Purpose of the Guidelines}

The overall goals of the guidelines were to provide recommendations on the amount of time in a 24-hour day that young children $(<5$ y) should spend being physically active or sleeping for their health and well-being, and to provide the maximum recommended time these children should spend on screen-based sedentary activities or restrained. With this guideline, WHO can address the gap in the recommendations on physical activity as children less than 5 years were not included in their Global Recommendations on Physical Activity for Health in 2010, ${ }^{3}$ and this will also contribute to the implementation of its recommendations by the Commission on Ending Childhood Obesity. ${ }^{4}$ In addition, these guidelines contribute to the broader Nurturing Care Framework for early childhood development. ${ }^{7}$ Nurturing care encompasses health, nutrition, and safety needs as well as early learning opportunities. The guidelines do not aim to address all aspects of early childhood development, but rather contribute to this broader context through recommendations specifically on physical activity, sedentary screen time, and time spent restrained or sitting and sleeping. 


\section{Target Audience}

The key audiences for the guidelines are as follows:

(1) Policymakers in ministries of health, education, and /or social welfare, working in high- as well as low- and middle-income countries, who formulate country-specific guidelines and who plan family, childcare, or community-based intervention programs.

(2) Persons working in nongovernmental organizations and early childhood development services who can use the guideline to define critical elements of childcare services.

(3) Those providing advice and guidance to caregivers (such as community/family nurses or doctors, pediatricians, or occupational therapists) can use the guideline to inform the content of their advice on these topics.

In order to inform parents and caregivers of the recommendations, derivative information products, guides, and tools should be developed at national level to ensure that the recommendations are disseminated using appropriate examples and infographics.

\section{Guideline Development Process}

The WHO Guidelines Review Committee approved the planning proposal from the internal steering group for the development of these guidelines in September 2017. A Guideline Development Group (GDG) was formed, consisting of a broad group of relevant experts in the field and end users of, and persons affected by, the recommendations, in accordance with the WHO Handbook for Guideline Development. ${ }^{8}$

In the first meeting (November 27-28, 2017), the GDG decided on the PICO (Population, Intervention [Exposure], Comparison, Outcome) questions, reviewed the existing systematic reviews, and identified the required updates. In their second meeting (April 18-20, 2018), the GDG reviewed the updated evidence and agreed on final recommendations by consensus.

\section{Systematic Reviews}

Four systematic reviews conducted up to April 20169-12 were updated through to March 2017, using the same search criteria and methods. ${ }^{13}$ The GDG reviewed the existing systematic reviews and requested that these be updated to include high-quality studies (experimental or longitudinal studies) published since the update and those identified in all official WHO languages to reflect the final PICO questions. Additional literature searches were conducted using the same search terms and methods as the original systematic reviews in French and Spanish, Arabic, Chinese, and Russian. Summaries of the evidence and Grading of Recommendations Assessment, Development and Evaluation (GRADE) tables were updated in December 2017. ${ }^{14}$

\section{Evidence to Recommendations}

The GDG used the GRADE Evidence to Decision framework for generating question-specific recommendations. The Evidence to Decision framework is a systematic, structured, and transparent approach to decision making. The framework uses explicit criteria for generating guideline recommendations in light of research evidence; certainty of evidence; caregiver and children's values and preferences; the balance between benefits and harms; and the impact of the recommendation on gender, social, and health equity, as well as the acceptability, feasibility, and resource implications of recommendations.

\section{Assessment of the Quality of Evidence}

Using the GRADE framework, the GDG examined the quality of primary research contributing to each outcome identified in the PICOs and assessed the overall quality of evidence taking into consideration the risk of bias, inconsistency, imprecision, indirectness of the evidence, and publication bias across each outcome. GRADE tables detailing the information for each PICO are available in the Web Annex Evidence Profiles (available online at https://apps.who.int/iris/handle/10665/311663).

The GDG considered reduced adiposity (body mass index-forage or other measures of childhood overweight or obesity); increased motor skills; and improvement in the measures of cognitive development, psychosocial health, and emotional regulation as favorable or desirable health outcomes and increased adiposity (body mass index-for-age or other measures of childhood overweight or obesity); decreased motor skills; and deterioration in the measures of cognitive development, psychosocial health, and emotional regulation as unfavorable or undesirable outcomes.

\section{Values and Preferences}

The GDG considered the values and preferences of those affected by the guidelines (in this case, parents and caregivers including those working with young children). Stakeholder surveys and focus group discussions undertaken in Canada and Australia ${ }^{13,15}$ indicated that there was low variability in parents' and stakeholders' preference for similar recommendations. Informants of the focus group discussions included those from vulnerable communities. ${ }^{13,15}$ No other evidence was available on values and preferences of stakeholders on physical activity, sedentary, and sleep behaviors in this age group. The Consensus Panel developing guidelines on movement behaviors for the 0 - to 5year age group in South Africa discussed early results from an assessment of children's physical activity, sedentary behavior, and sleep patterns. These data formed part of postgraduate dissertations and are in the process of being prepared for publication, but the data were shared with the GDG during the second GDG meeting. All these sources of information were used to guide the GDG's discussion on values and preferences, in addition to expert knowledge from the GDG on the situation in their settings.

\section{Resource Implications}

A systematic review of the resource implications for this guideline was prepared by Olga Milliken of the WHO Collaborating Centre on Chronic Non-Communicable Disease Policy, Ottawa, Canada. This review considered the evidence for physical activity interventions and concluded that there was limited evidence on costeffectiveness of relevant interventions for children and no evidence for children less than 5 years and their parents.

The expert opinion of the GDG was that the cost to government and nongovernment organizations of guideline implementation would be minimal if recommended physical activity could be relatively easily incorporated by individuals (parents and their children) into their lives, or existing resources in primary care, day-care/school-based settings can be shifted resulting in increased physical activity. No evidence was available on the cost to parents or caregivers for implementing the recommendations. 


\section{Equity, Acceptability, and Feasibility}

The GDG concluded that adhering to the integrated 24-hour movement guidelines during the early years is likely to benefit all groups equally, and recommendations could be achieved equitably. ${ }^{13,15}$

\section{Peer Review and Final Approval}

The draft guidelines were reviewed by 5 external peer reviewers identified by the GDG and Steering Group, who provided relevant expertise, including in program implementation and represented 5 WHO regions. The final guidelines and recommendations were reviewed and approved by the Guidelines Review Committee in February 2019, and the guidelines were published and launched in April 2019, with extensive media coverage and interest.

\section{Results}

Additional literature searches to update the existing systematic reviews yielded the following: for physical activity, 15 additional studies were identified, of which only 6 were of experimental or longitudinal design and were extracted; for sedentary behavior, an additional 15 studies were identified, of which only 4 were longitudinal studies (no experimental studies) and that were extracted; for sleep, an additional 11 studies were identified, of which only 5 were of longitudinal study design and were extracted; and for integrated physical activity, sedentary, and sleep (movement) behaviors, an additional 4 studies were identified, of which 3 were of experimental or longitudinal design and were extracted.

\section{Summary of Recommendations}

Full details of the GRADE tables and Evidence to Decision framework used in development of the WHO guidelines are available at https://apps.who.int/iris/handle/10665/311663.

For the greatest health benefits, infants and children should meet all the recommendations for physical activity, sedentary behavior, and sleep in a 24-hour period, as shown in Figure 1.

In a 24-hour day, infants ( $<1$ y) should

- be physically active several times a day in a variety of ways, particularly through interactive floor-based play; more is better. For those not yet mobile, this includes at least 30 minutes in prone position (tummy time) spread throughout the day while awake;

- not be restrained for more than 1 hour at a time (eg, prams/ strollers, high chairs, or strapped on a caregiver's back). Screen time is not recommended. When sedentary, engaging in reading and storytelling with a caregiver is encouraged; and

- have 14 to 17 hours (0-3 mo of age) or 12 to 16 hours (4-11 mo of age) of good quality sleep, including naps.

In a 24-hour day, children 1-2 years of age should

- spend at least 180 minutes in a variety of types of physical activities at any intensity, including moderate- to vigorousintensity physical activity, spread throughout the day; more is better;

- not be restrained for more than 1 hour at a time (eg, prams/ strollers, high chairs, or strapped on a caregiver's back) or sit for extended periods of time. For 1-year-old children,
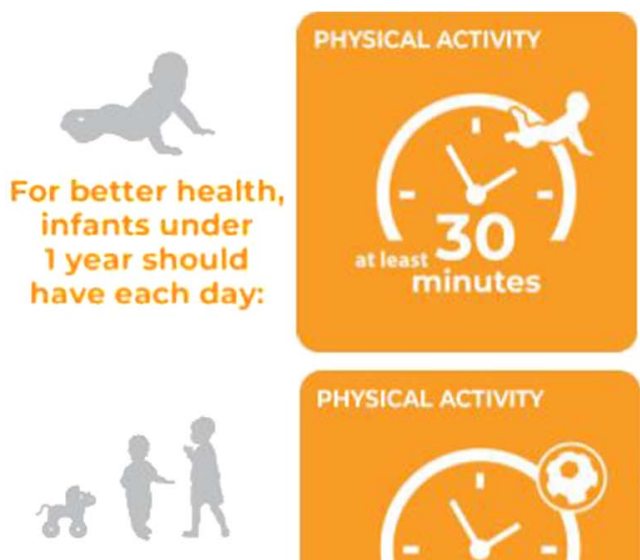

Children $1-2$ years
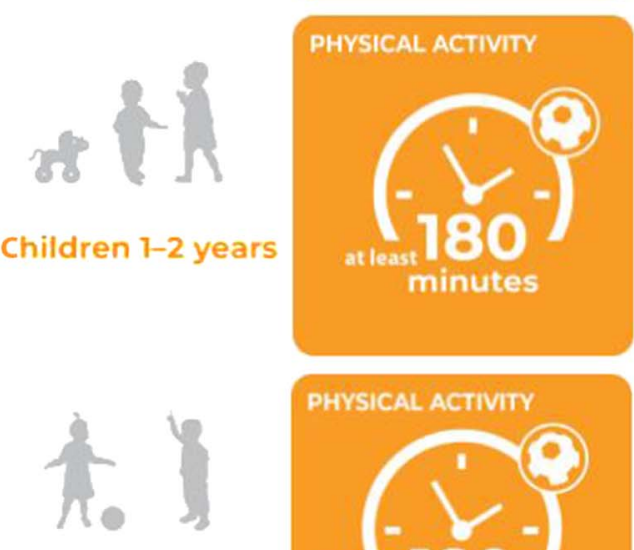

Children $3-4$ years

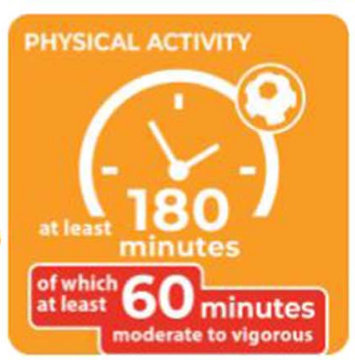

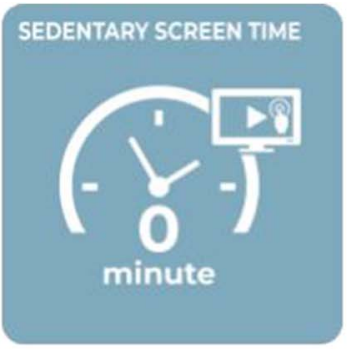
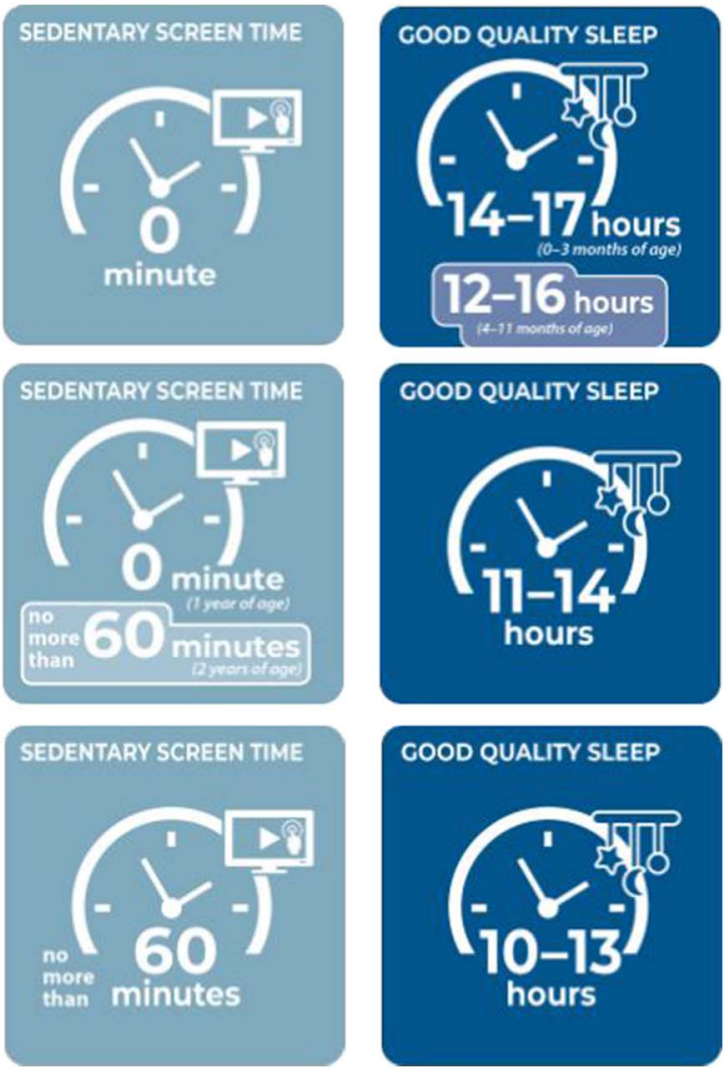

Figure 1 - Summary of recommendations. 
sedentary screen time (such as watching TV or videos, playing computer games) is not recommended. For those aged 2 years, sedentary screen time should be no more than 1 hour; less is better. When sedentary, engaging in reading and storytelling with a caregiver is encouraged; and

- have 11 to 14 hours of good quality sleep, including naps, with regular sleep and wake-up times.

In a 24-hour day, children 3-4 years of age should

- spend at least 180 minutes in a variety of types of physical activities at any intensity, of which at least 60 minutes is moderate- to vigorous-intensity physical activity, spread throughout the day; more is better;

- not be restrained for more than 1 hour at a time (eg, prams/ strollers) or sit for extended periods of time. Sedentary screen time should be no more than 1 hour; less is better. When sedentary, engaging in reading and storytelling with a caregiver is encouraged; and

- have 10 to 13 hours of good quality sleep, which may include a nap, with regular sleep and wake-up times.

For the greatest health benefits, infants and young children should meet all the recommendations for physical activity, sedentary behavior, and sleep in a 24-hour period.

Replacing restrained or sedentary screen time with moderateto vigorous-intensity physical activity, while preserving sufficient sleep can provide additional health benefits. ${ }^{16}$

\section{Discussion}

WHO, as the specialized health agency of the United Nations, has a specific role in developing normative guidance and recommendations for both clinical and public health practice. In doing so, WHO undertakes a rigorous process for guidelines development and in particular to meet the needs of low- and middle-income countries that may not have the resources to develop national guidelines de novo. These guidelines and a summary brochure have been translated into all the official languages of WHO, social media infographics were prepared and shared during the launch, and additional materials have been made available to disseminate information on the guidelines. A set of standards for physical activity, sedentary behavior, and sleep in early childhood education and care settings is under development, and WHO provides support to countries wishing to adopt and adapt the guidelines for national implementation. The guidelines have also been incorporated into work plans for the Nurturing Care Framework for early childhood development. WHO is currently updating the 2010 Global Recommendations on Physical Activity for Health, with a view to the current guidelines for children less than 5 years of age forming part of a complete life course approach to physical activity. The guidelines also highlight the need for improved surveillance of physical activity in young children, such that global comparable estimates of physical activity and sedentary behavior in this age group may be tracked to monitor progress.

\section{Conclusions}

The first global guidelines on physical activity, sedentary behavior, and sleep for children less than 5 years of age were published in April 2018 and are available at https://apps.who.int/iris/handle/ 10665/311663. Implementation of the guidelines should be a major element of the national and international response to the global epidemic of childhood obesity, and adherence to the guideline will improve many aspects of child health and development and improve long-term health.

\section{Acknowledgments}

The authors acknowledge the dedicated support and guidance of the Guidelines Review Committee Secretariat at WHO and thank the Guidelines Steering Group, Development Group, and Peer Reviewers for their contributions to this process. The second meeting of the GDG was made possible with the financial support of the Public Health Agency of Canada.

\section{References}

1. Guthold R, Stevens GA, Riley LM, Bull FC. Worldwide trends in insufficient physical activity from 2001 to 2016: a pooled analysis of 358 population-based surveys with 1.9 million participants. Lancet Glob Health. 2018;6(10):e1077-e1086. PubMed ID: 30193830 doi:10.1016/S2214-109X(18)30357-7

2. World Health Organization. Global Status Report on Noncommunicable Diseases. Geneva, Switzerland: World Health Organization; 2014.

3. World Health Organization. Global Recommendations on Physical Activity for Health. Geneva, Switzerland: World Health Organization; 2010.

4. Commission on Ending Childhood Obesity. Report of the Commission on Ending Childhood Obesity. Geneva, Switzerland: World Health Organization; 2016.

5. Janz KF, Burns TL, Levy SM; Iowa Bone Development Study. Tracking of activity and sedentary behaviors in childhood: the Iowa Bone Development Study. Am J Prev Med. 2005;29(3):171-178. PubMed ID: 16168865 doi:10.1016/j.amepre.2005.06.001

6. World Health Organization. Global Action Plan on Physical Activity 2018-2030: More Active People for a Healthier World. Geneva, Switzerland: World Health Organization; 2018.

7. World Health Organization, United Nations Children's Fund, World Bank Group. Nutruring Care for Early Childhood Development: A Framework for Helping Children Survive and Thrive to Transform Health and Human Potential. Geneva, Switzerland: World Health Organization; 2018.

8. World Health Organization. WHO Handbook for Guideline Development. 2nd ed. Geneva, Switzerland: World Health Organization; 2014.

9. Carson V, Lee EY, Hewitt L, et al. Systematic review of the relationships between physical activity and health indicators in the early years (0-4 years). BMC Public Health. 2017;17(suppl 5):854. PubMed ID: 29219090 doi:10.1186/s12889-017-4860-0

10. Chaput JP, Gray CE, Poitras VJ, et al. Systematic review of the relationships between sleep duration and health indicators in the early years (0-4 years). BMC Public Health. 2017;17(suppl 5):855. PubMed ID: 29219078 doi:10.1186/s12889-017-4850-2

11. Kuzik N, Poitras VJ, Tremblay MS, Lee EY, Hunter S, Carson V. Systematic review of the relationships between combinations of movement behaviours and health indicators in the early years (0-4 years). BMC Public Health. 2017;17(suppl 5):849. PubMed ID: 29219071 doi:10.1186/s12889-017-4851-1

12. Poitras VJ, Gray CE, Janssen X, et al. Systematic review of the relationships between sedentary behaviour and health indicators in the early years (0-4 years). BMC Public Health. 2017;17(suppl 5): 868. PubMed ID: 29219092 doi:10.1186/s12889-017-4849-8 
13. Okely AD, Ghersi D, Hesketh $\mathrm{KD}$, et al. A collaborative approach to adopting/adapting guidelines - the Australian 24-hour movement guidelines for the early years (Birth to 5 years): an integration of physical activity, sedentary behavior, and sleep. BMC Public Health. 2017;17(suppl 5):869. PubMed ID: 29219094 doi:10.1186/s12889-017-4867-6

14. World Health Organization. Summary Report of the Update of Systematic Reviews of the Evidence to Inform the WHO Guidelines on Physical Activity, Sedentary Behaviour and Sleep in Children Under 5 Years of Age. Geneva, Switzerland: World Health Organization; 2018.
15. Riazi N, Ramanathan S, O’Neill M, Tremblay MS, Faulkner G. Canadian 24-hour movement guidelines for the early years ( $0-4$ years): exploring the perceptions of stakeholders and end users regarding their acceptability, barriers to uptake, and dissemination. BMC Public Health. 2017;17(suppl 5):841. PubMed ID: 29219087 doi:10.1186/ s12889-017-4853-z

16. World Health Organization. Guidelines on Physical Activity, Sedentary Behaviour and Sleep for Children Under 5 Years of Age. Geneva, Switzerland: World Health Organization; 2019. 